\title{
Alterações na dimensão transversal pela expansão rápida da maxila
}

Roberto M. A. Lima Filho*

\begin{abstract}
Resumo
As deformidades transversais, que se manifestam tipicamente pela mordida cruzada unilateral ou bilateral, são os problemas esqueléticos que mais sequelas podem causar na região craniofacial. Entretanto, são as deformidades que melhor se adaptam às alterações ortopédicas. A expansão rápida da maxila tornou-se rotina na prática ortodôntica. Embora inicialmente tenha sido utilizada na correção da mordida cruzada posterior, atualmente sua indicação ampliou-se para a expansão indireta do arco inferior, obtenção de espaço para correção de apinhamento dentário, correção axial dos dentes posteriores, melhora na estética do sorriso e auxílio no tratamento de pacientes Classe II. A expansão ortopédica da maxila vem atraindo cada vez mais a atenção da comunidade científica devido, principalmente, à sua aplicação e capacidade de alterar o crescimento craniofacial em diversas situações clínicas. Esse tipo de intervenção ortopédica possui grande utilidade terapêutica, pois sua aplicação em diversas anormalidades apresenta maior versatilidade quando comparada aos aparelhos de modificação de crescimento disponiveis atualmente para o tratamento ortodôntico.
\end{abstract}

Palavras-chave: Dimensão transversal. Expansão rápida da maxila. Má oclusão Classe II.

As deformidades transversais fazem parte das alterações dentofaciais encontradas pelo ortodontista. Dentre elas, a mais comum é a deficiência maxilar transversal. O tratamento comumente empregado nesses casos é a expansão rápida da maxila (ERM) - método de alteração ortopédica do crescimento craniofacial utilizado em diversas situações clínicas. Esse procedimento consiste na aplicação de força à maxila através dos dentes, expandindo a sutura palatina mediana e, consequentemente, a maxila.

Embora essa deformidade seja um dos problemas esqueléticos que mais sequelas pode causar na região craniofacial, é também a que melhor se adapta às alterações ortopédicas, quando comparada às outras regiões do complexo craniofacial, após o tratamento.

As deficiências maxilares transversais observadas pelo clínico em pacientes que buscam tratamento ortodôntico manifestam-se, tipicamente, pela mordida cruzada unilateral ou bilateral (Fig. 1). Existem casos com essa deficiência que não apresentam mordida cruzada posterior, nos quais a oclusão é aparentemente normal, mas há compensação pela inclinação axial dos dentes superiores para vestibular e dos inferiores para lingual.

As deficiências maxilares transversais, se não identificadas no exame clínico e não definidas no diagnóstico, conduzem a diversas manifestações

\footnotetext{
* Doutor em Ortodontia pela UFRJ. Pós-graduado em Ortodontia pela Universidade de Illinois em Chicago - EUA. Diplomado pelo American Board of Orthodontics. Membro da Edward H. Angle Society of Orthodontists. Presidente Fundador do Board Brasileiro de Ortodontia e Ortopedia Facial.
} 
encontradas no dia a dia, como hipoplasia maxilar, crescimento facial assimétrico, posicionamento e desvio funcional da mandíbula, estética dentofacial comprometida, respostas periodontais adversas e outros problemas funcionais decorrentes da constrição maxilar.

Nas últimas duas décadas, a expansão rápida da maxila tornou-se rotina na prática ortodôntica. Embora esse procedimento tenha sido inicialmente utilizado para a correção da mordida cruzada posterior, existem diversas aplicações para essa técnica, tais como a expansão indireta do arco inferior, a obtenção de espaço para a correção de apinhamento dentário, a correção axial dos dentes posteriores, a melhora na estética do sorriso e o auxílio no tratamento de pacientes Classe II.

\section{EXPANSÃO INDIRETA DO ARCO INFERIOR}

A ERM tem sido usada por mais de um século para o tratamento da constrição maxilar e, embora existam muitas publicações sobre esse método, praticamente todas tratam de reações que ocorrem no complexo maxilar. Muito pouco tem
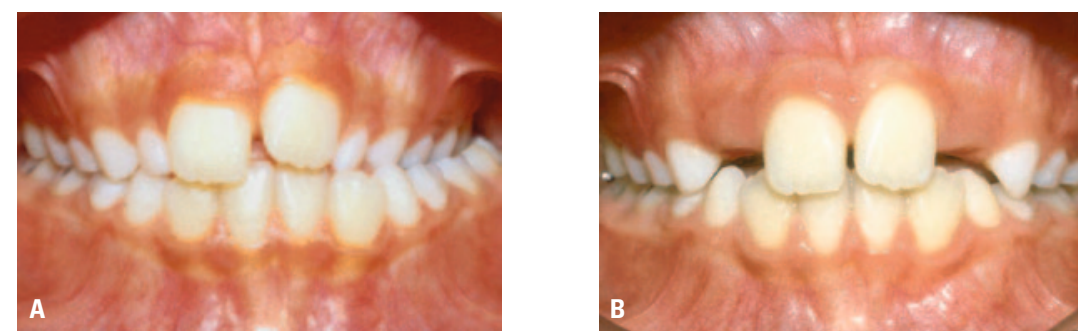

FIGURA 1 - Paciente portador de deficiência maxilar transversal: A) mordida cruzada unilateral na fase pré-tratamento, B) normalização da dimensão transversal após a expansão rápida da maxila.
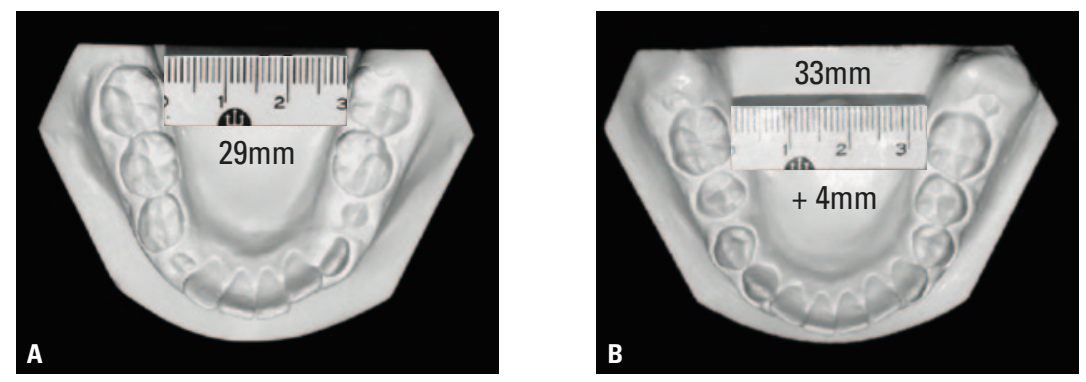

FIGURA 2 - Modelos de estudo do arco inferior de paciente com discrepância transversal: A) pré-tratamento, B) progresso evidenciando a expansão espontânea de 4mm na distância intermolares após a expansão rápida da maxila, sem uso de aparelho no arco inferior. 
Haas $^{10}$, investigando correções ortopédicas e ortodônticas de dez pacientes tratados pela ERM, comprovou uma estabilidade de 4 a $5 \mathrm{~mm}$ na expansão da distância intercaninos do arco inferior após vários anos sem contenção; e de 9 a $12 \mathrm{~mm}$ na expansão dos dentes posterossuperiores. Sandstrom, Klapper e Papaconstantinou ${ }^{24}$, avaliando o efeito da ERM no arco inferior em 28 pacientes que utilizaram o expansor tipo Haas, concluíram que o aumento médio na distância intercaninos, mantido dois anos pós-contenção, foi de $1,1 \mathrm{~mm}$ ganho estatisticamente significativo em relação à distância inicial. Lima, Lima, Lima Filho e Oyen ${ }^{20}$, estudando pacientes Classe I tratados exclusivamente com ERM durante a dentadura mista, constataram uma expansão espontânea no arco inferior e notável estabilidade clínica a longo prazo.

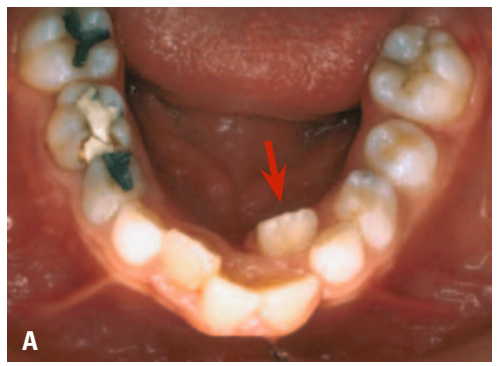

\section{MAXILA VERSUS MANDÍBULA}

Problemas associados com a posição dos dentes na mandíbula são mais difíceis de tratar clinicamente, uma vez que a estrutura mandibular é altamente resistente e, portanto, menos suscetível às forças ortodônticas e ortopédicas aplicadas durante o tratamento. Movimentos dentários, com exceção do deslocamento de dentes em locais de extração, limitam-se principalmente às inclinações ou às rotações, sendo influenciados por diferenças na estrutura óssea entre o processo alveolar e a base apical mandibular.

$\mathrm{O}$ arco dentário superior difere do inferior porque a maxila é composta por dois ossos unidos nas suturas palatina mediana e intermaxilares (Fig. 4). Essa característica anatômica possibilita que o efeito ortopédico na maxila seja mais eficaz.

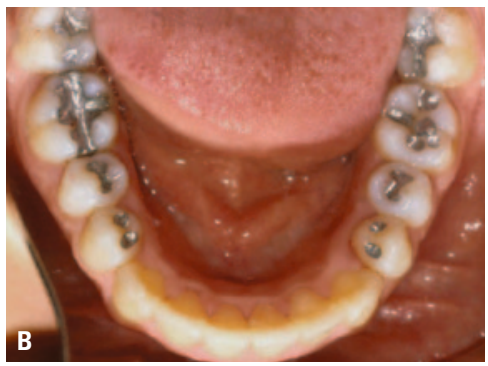

FIGURA 3 - Paciente com acentuada falta de espaço no arco inferior: A) pré-tratamento, onde se nota a posição lingual do incisivo lateral inferior esquerdo devido à falta de espaço (seta), B) pós-tratamento mostrando a verticalização dos dentes posteriores e a obtenção de espaço para 0 alinhamento dentário.
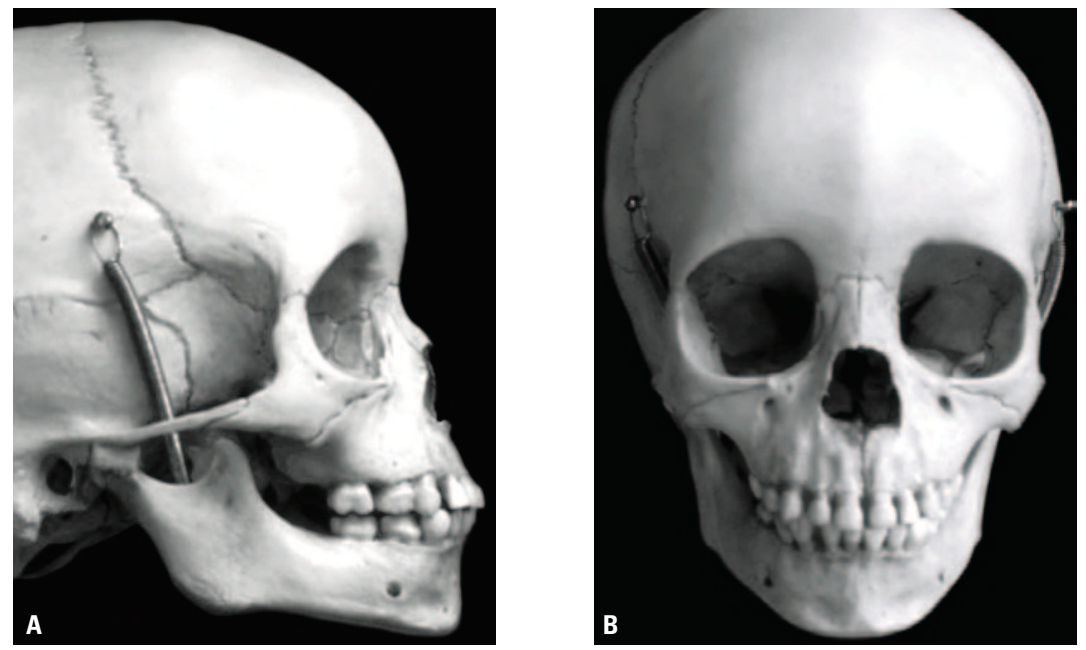

FIGURA 4 - Crânio de criança em norma lateral (A) e norma frontal (B). Note as suturas intermaxilares. 
Por meio da ERM, é possível posicionar as bases apicais maxilar e mandibular em equilíbrio, restabelecendo com menor esforço a boa oclusão funcional. Infelizmente, não existe técnica similar para a mandíbula, devido à fusão da linha de sutura durante o primeiro ano após o nascimento.

\section{CORREÇÃO DE APINHAMENTO DENTÁRIO}

O apinhamento dentário pode ser definido como uma discrepância na relação entre o tamanho dos dentes e o tamanho dos maxilares, resultando em quebra de contato e rotação dos dentes.

Howe, McNamara e O'Connor ${ }^{12}$, estudando 50 pacientes com apinhamento e 54 sem, verificaram que não houve diferença significativa no tamanho dos dentes entre os grupos. Por outro lado, esse estudo revelou diferença significativa de 5 a $6 \mathrm{~mm}$ na largura do arco. Esses resultados indicam que o tamanho e o perímetro do arco parecem ser mais importantes, na etiologia do apinhamento dentário, do que o tamanho dos dentes.

A dimensão transversal da maxila pode ser expandida e o espaço temporário na região da sutura palatina mediana é preenchido por novo tecido ósseo. As fibras transeptais que ligam os incisivos centrais superiores induzem a movimentação desses dentes, resultando no fechamento do diastema produzido durante a expansão (Fig. 5). Com essas alterações, ocorre aumento no comprimento total do arco, possibilitando a acomodação no arco dentário de dentes girados, deslocados ou impactados (Fig. 6). Adkins, Nanda e Currier ${ }^{1}$, examinando
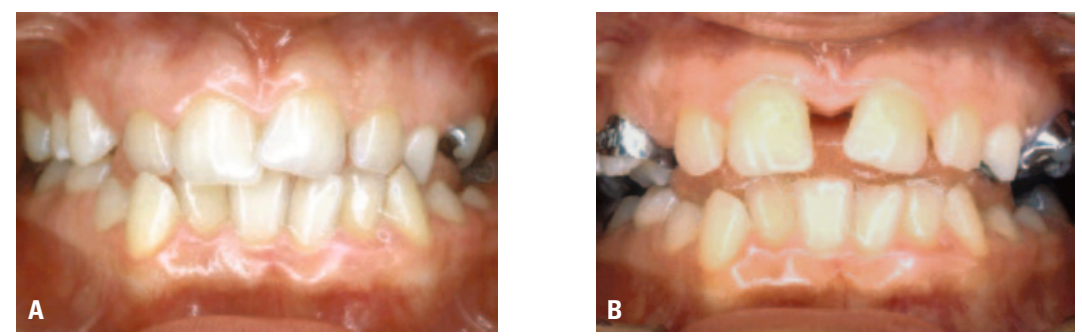

FIGURA 5 - Paciente portador de deficiência maxilar transversal: A) pré-tratamento e B) pós-expansão. 0 fechamento do diastema na linha média propiciará espaço para a correção do apinhamento dentário.
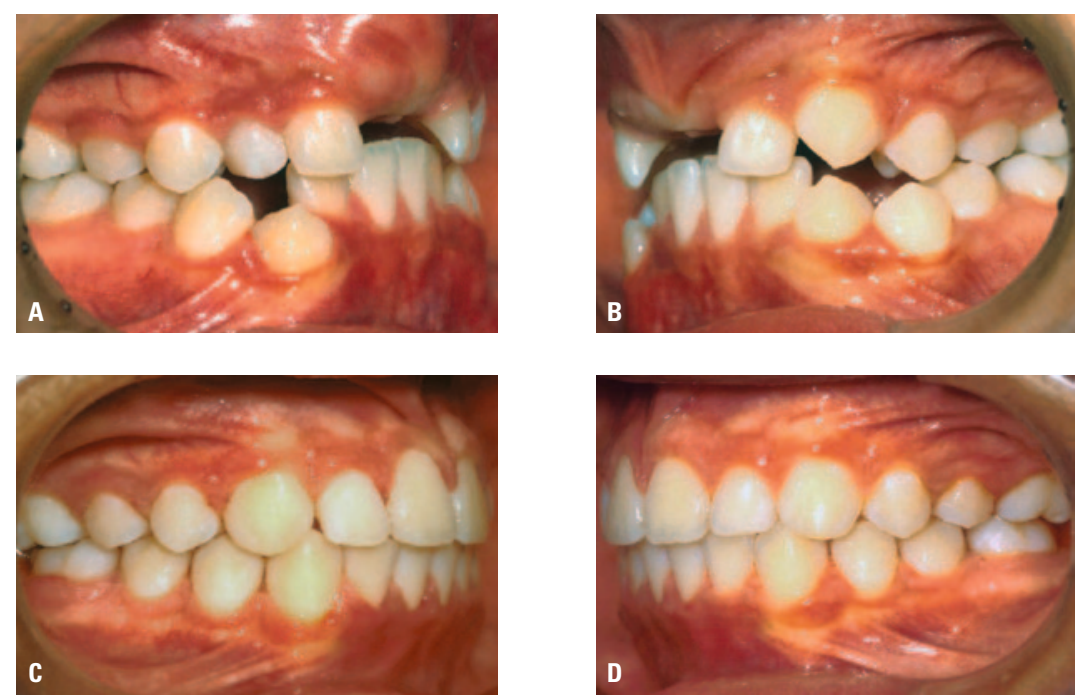

FIGURA 6 - Paciente portador de má oclusão de Classe I: A, B) falta acentuada de espaço na fase pré-tratamento e C, D) oclusão obtida após a ERM e 0 tratamento corretivo. 
a relação entre a expansão rápida da maxila e as mudanças no perímetro do arco, constataram que cada milímetro de aumento na largura transpalatina na região de pré-molares produz $0,7 \mathrm{~mm}$ de aumento no perímetro do arco.

No início da dentadura mista, a expansão maxilar aumenta a largura transversal do osso basal da maxila, resultando na correção do apinhamento dentário e diminuindo a necessidade de extração de dentes permanentes ${ }^{23}$ (Fig. 6).
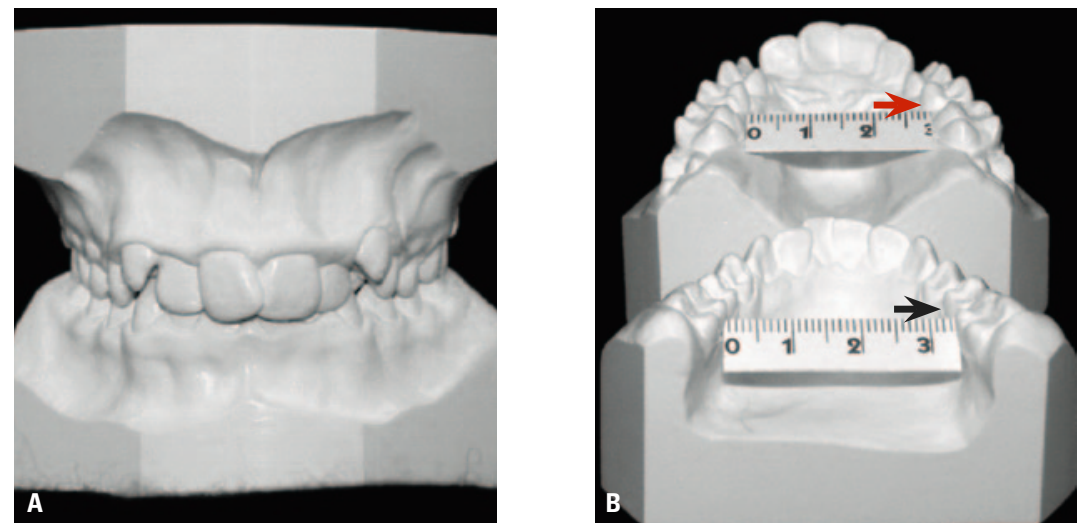

FIGURA 7 - Modelos de estudo de paciente portadora de má oclusão de Classe II na fase pré-tratamento: A) vista frontal e B) vista póstero-oclusal mostrando deficiência maxilar e inclinação desfavorável dos dentes posteriores superiores inclinados para vestibular (seta vermelha) e inferiores para lingual (seta preta).

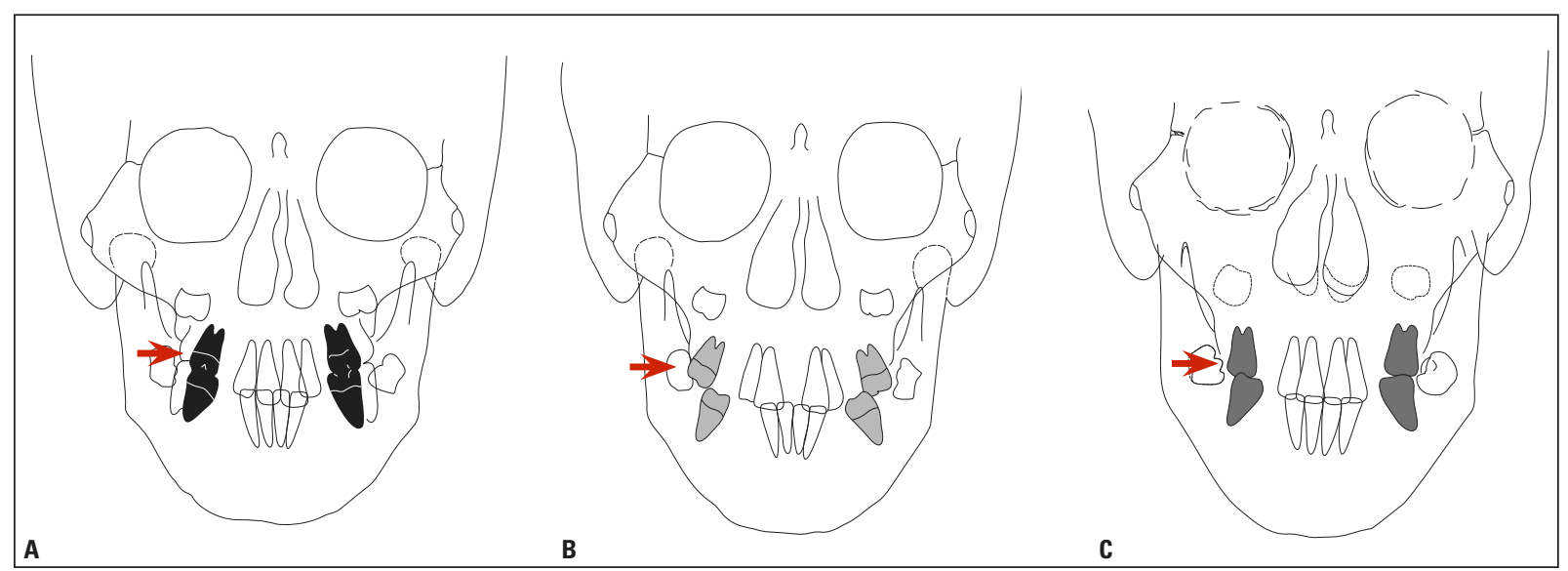

FIGURA 8 - Traçados cefalométricos posteroanteriores de paciente Classe II: A) na fase pré-tratamento nota-se uma inclinação desfavorável dos dentes posteriores (seta), B) na fase pós-expansão observa-se a sobrecorreção dos dentes posterossuperiores (seta), e C) na fase pós-tratamento nota-se a correção da inclinação axial desfavorável dos dentes posteriores (seta). 
Corrigindo-se apenas a mordida cruzada dentária não se atinge o objetivo principal do tratamento ortopédico, que é estabelecer uma relação mais favorável entre as bases dentárias ${ }^{21}$. Quando isso ocorre, os dentes posterossuperiores devem sempre estar sobre-expandidos, produzindo tendência à mordida cruzada vestibular. A relação ideal é manter contato entre as cúspides linguais dos dentes posterossuperiores com as cúspides vestibulares dos posteroinferiores.

A sobrecorreção (Fig. 9) é fundamental, pois, após a expansão, os dentes posterossuperiores apresentam vários graus de inclinação, devido à compressão e distensão do ligamento periodontal e também ao dobramento dos processos alveolares. A estabilidade somente é atingida por meio de neoformação óssea nas suturas, pois a expansão obtida pelo dobramento alveolar e deslocamento dentário está sujeita à total recidiva. $\mathrm{Na}$ correção da mordida cruzada posterior, pouca sobrecorreção induz à recidiva, uma vez que não foi reservada margem para o retorno dos dentes inclinados.

\section{ESTÉTICA DO SORRISO}

Em casos de deficiência maxilar transversal, a projeção dentária lateral é insuficiente para preencher os cantos do sorriso, ou seja, a dentição não se estende até as comissuras labiais (Fig. 10).

Sarver ${ }^{25}$ empregou o termo espaço negativo para descrever as sombras que aparecem nos cantos da boca, durante o sorriso, em pacientes portadores de maxila estreita e cônica. Esse conceito, em termos protéticos, é chamado de corredores bucais. Não é seguro extrair dentes para resolver o problema de apinhamento em pacientes com espaço negativo, pois a possibilidade de melhorar o problema estético seria diminuída.

O tratamento indicado inclui a ERM, que aumenta consideravelmente a largura e o comprimento dos arcos superior e inferior e, por conseguinte, substitui o espaço negativo por estrutura dentoalveolar ${ }^{16,25}$ (Fig. 11).

A expansão rápida da maxila para resolver problemas estéticos (sorriso) deve ser reconhecida como indicação para pacientes portadores de deficiência maxilar transversal e arcos dentários estreitos. Além disso, a avaliação do sorriso poderia constar no protocolo de avaliação para diagnóstico de anormalidades dentofaciais.

\section{CLASSE II E DIMENSÃO TRANSVERSAL}

A morfologia facial é determinada, em grande parte, pela posição relativa da maxila e da mandíbula antes, durante e após o pico puberal de crescimento. A posição harmoniosa entre as bases maxilares, em relação ao crânio, não somente é responsável por uma boa oclusão funcional, como também por uma estética facial agradável.
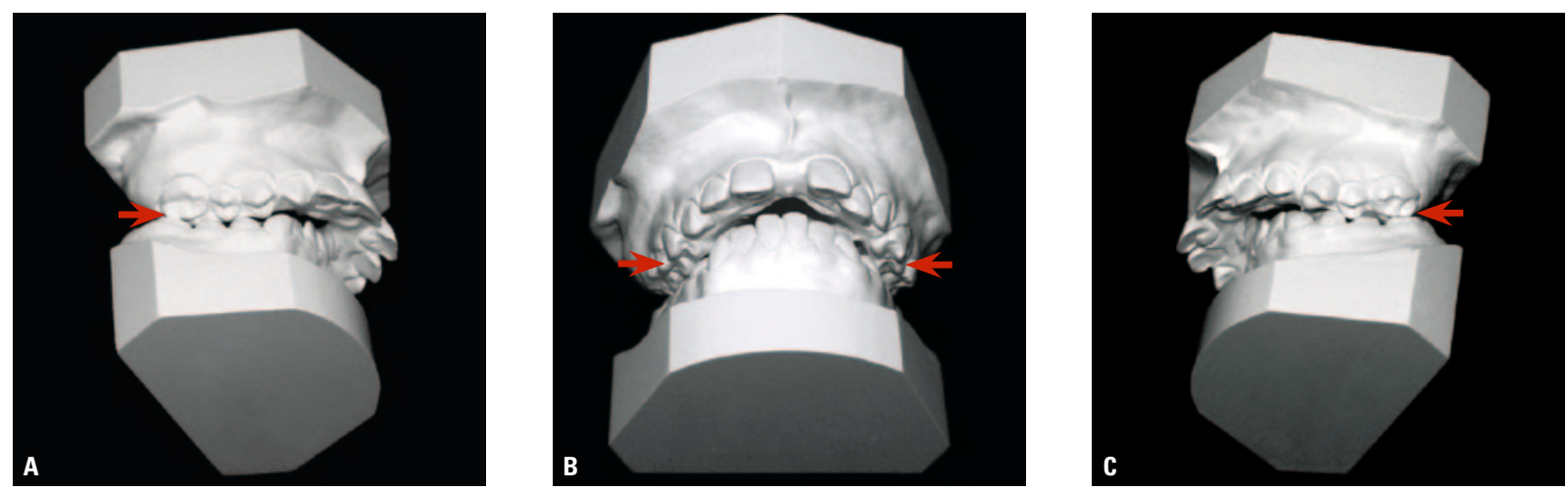

FIGURA 9 - Modelos de estudo de paciente portador de má oclusão de Classe II na fase pós-expansão: observa-se a sobrecorreção e as cúspides linguais dos dentes posterossuperiores em contato com as cúspides vestibulares dos posteroinferiores (setas). 

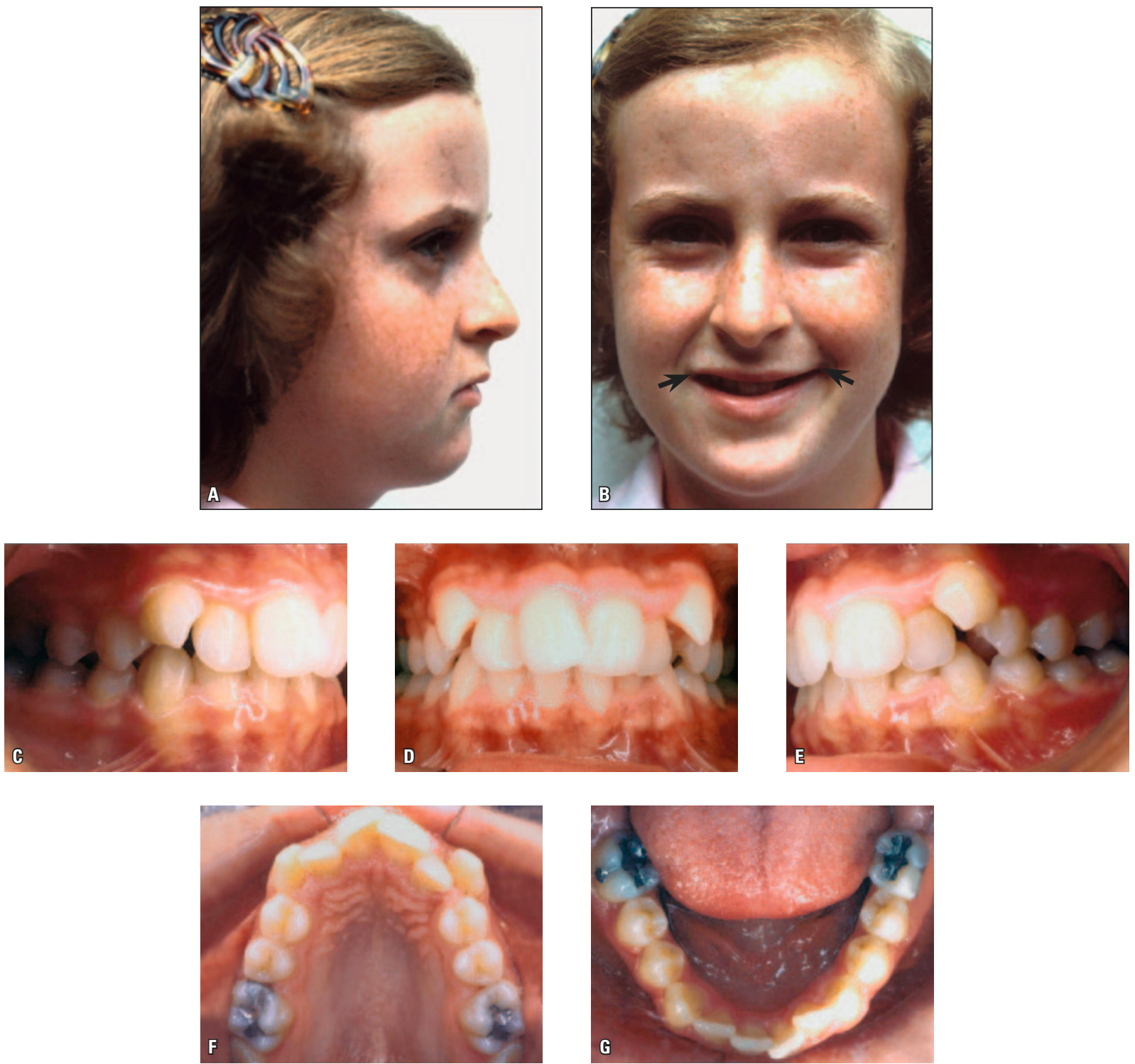

FIGURA 10 - Paciente portadora de má oclusão de Classe II, aos 11 anos e 11 meses de idade na fase pré-tratamento. A, B) Perfil reto e espaços escuros nas extremidades dos lábios (setas). C, D, E) Vistas intrabucais direita, frontal e esquerda mostrando a relação de Classe II. F, G) Vistas intrabucais oclusais evidenciando arcos estreitos e com falta de espaço.

Quando as proporções entre a maxila e a mandíbula estão alteradas entre si, ou em relação ao crânio, observa-se deformidades dentofaciais. Essas deformidades podem ocorrer, na maxila e/ou mandíbula, nas três dimensões do espaço, mas frequentemente ocorrem no plano anteroposterior, manifestando-se como má oclusão de Classe II.
Trabalhos recentes sugerem que a má oclusão de Classe II está relacionada à deficiência em largura da maxila ${ }^{6,15,18,26,27}$ (Fig. 12). Essa deficiência ocorre em crianças antes dos seis anos de idade, e a abertura da maxila atrésica é fundamental para a correção esquelética definitiva dessa má oclusão e prevenção de crescimento protrusivo² (Fig. 13). 

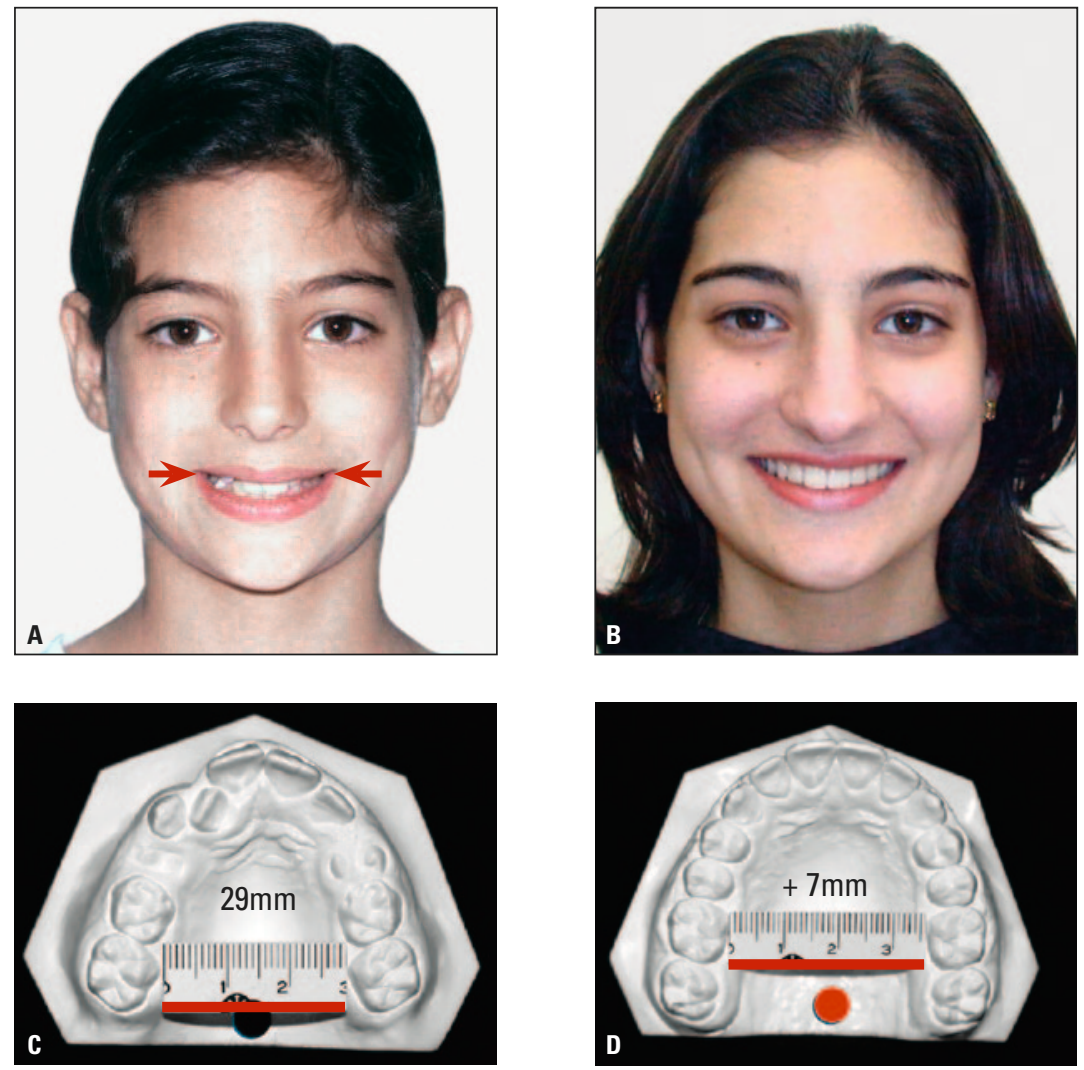

FIGURA 11 - Paciente portadora de deficiência maxilar transversal: A) vista facial sorrindo pré-tratamento, onde observa-se os espaços escuros nas extremidades dos lábios (setas); B) vista facial sorrindo pós-tratamento, com melhora na estética do sorriso devido à expansão transversal da maxila; modelos de estudo C) pré-tratamento e D) pós-tratamento.

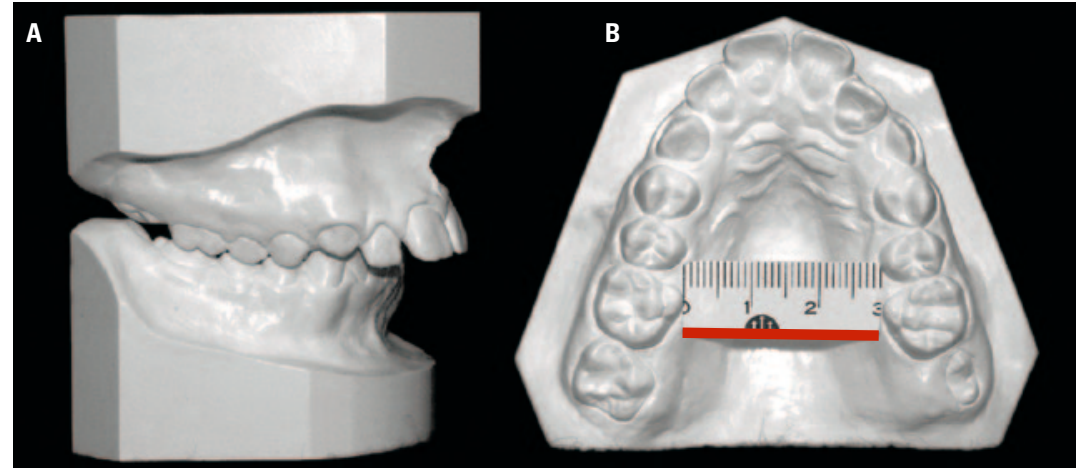

FIGURA 12 - Modelos de estudo de paciente portador de má oclusão de Classe II: A) vista lateral mostrando alterações anteroposterior e vertical, e B) vista oclusal superior mostrando a deficiência transversal.

A má oclusão de Classe II pode mascarar a deficiência maxilar transversal, pois os dentes posterossuperiores ocluem em uma região mais estreita da mandíbula, apresentando compensação em suas inclinações axiais ${ }^{28}$. Nesses casos, a expansão do arco maxilar promove uma tração muscular (bucofacial e mastigatória) na direção mais adequada, maximizando o crescimento da musculatura 

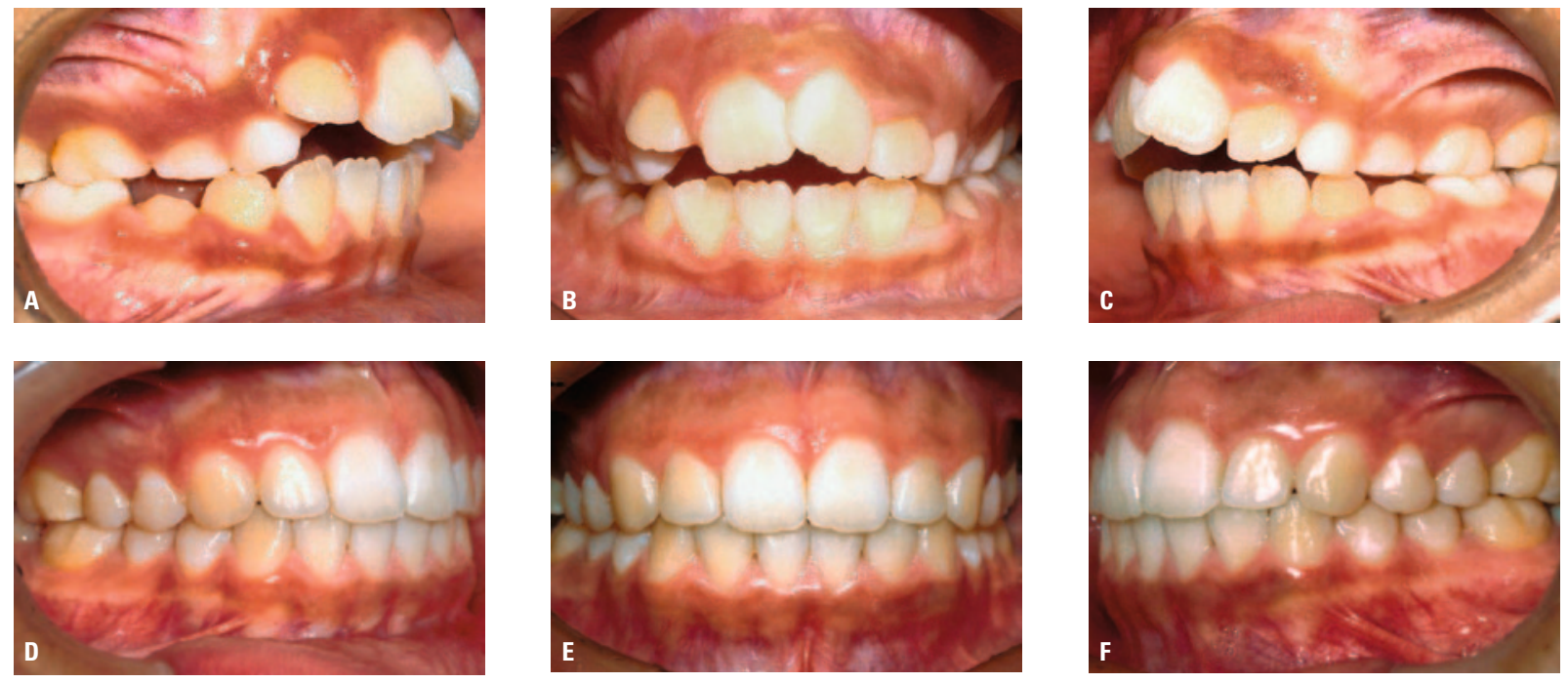

FIGURA 13 - Paciente portador de má oclusão de Classe II tratado com ERM: A, B, C) pré-tratamento e D, E, F) pós-tratamento.

bucofacial e proporcionando efeito favorável ao crescimento dos maxilares, alinhamento dentário e estética dentolabial. Expandindo o arco superior, pode-se criar uma excelente condição para que a mandíbula possa expressar seu pleno crescimento, auxiliando na correção da Classe II $^{19}$.

Em casos de Classe II divisão 1, o arco superior geralmente apresenta-se constricto ou em forma de $\mathrm{V}$, associado à sobremordida profunda, podendo resultar em força de deslocamento posterior na mandíbula. A abertura da base apical maxilar libera a mandíbula para mover-se anteriormente, visando a correção parcial da relação de Classe II molar. Casos de Classe II divisões 1 e 2 apresentam retrusão funcional da mandíbula, beneficiando-se, portanto, da liberação mandibular como resultado da expansão da maxila ${ }^{9}$.

A correção espontânea da Classe II pode ser observada em casos de discrepância leve ou moderada. A figura 14 ilustra a correção espontânea de uma discrepância anteroposterior após a ERM sem o uso de aparelhagem adicional.

O sucesso do tratamento da má oclusão de Classe II em crianças está relacionado ao aproveitamento do crescimento normal e ao potencial para interferir nesse crescimento por meio de mecânicas apropriadas ${ }^{5}$.

O aparelho extrabucal equipara-se a um método biológico e seguro de tratamento, muito útil para a correção da má oclusão de Classe II no final da dentadura mista ou início da permanente, que permite distalizar os dentes posteriores em locais de crescimento da maxila9,11. Se o tratamento das más oclusões de Classe II for iniciado durante a transição da dentadura, após a irrupção dos primeiros pré-molares, a correção pode ser bem sucedida em 90\% dos casos com esse tipo de má oclusão ${ }^{7}$.

Na correção da má oclusão de Classe II com aparelho extrabucal é essencial expandir a maxila estreita, para evitar a rotação da mandíbula no sentido horário ${ }^{17}$. Estudos indicam que a expansão da maxila pode ser obtida utilizando-se o aparelho extrabucal com arco interno expandido ${ }^{13,14}$.

Quando o arco interno do aparelho extrabucal é expandido, existe uma força expansiva sobre os molares que tende a fazer com que suas coroas girem para fora. Entretanto, essa tendência é compensada pela tração do elástico cervical ao arco externo dobrado para cima, o qual produz força lateral nas raízes dos molares ${ }^{9}$ (Fig. 15). Esse procedimento produz expansão lenta na base apical 

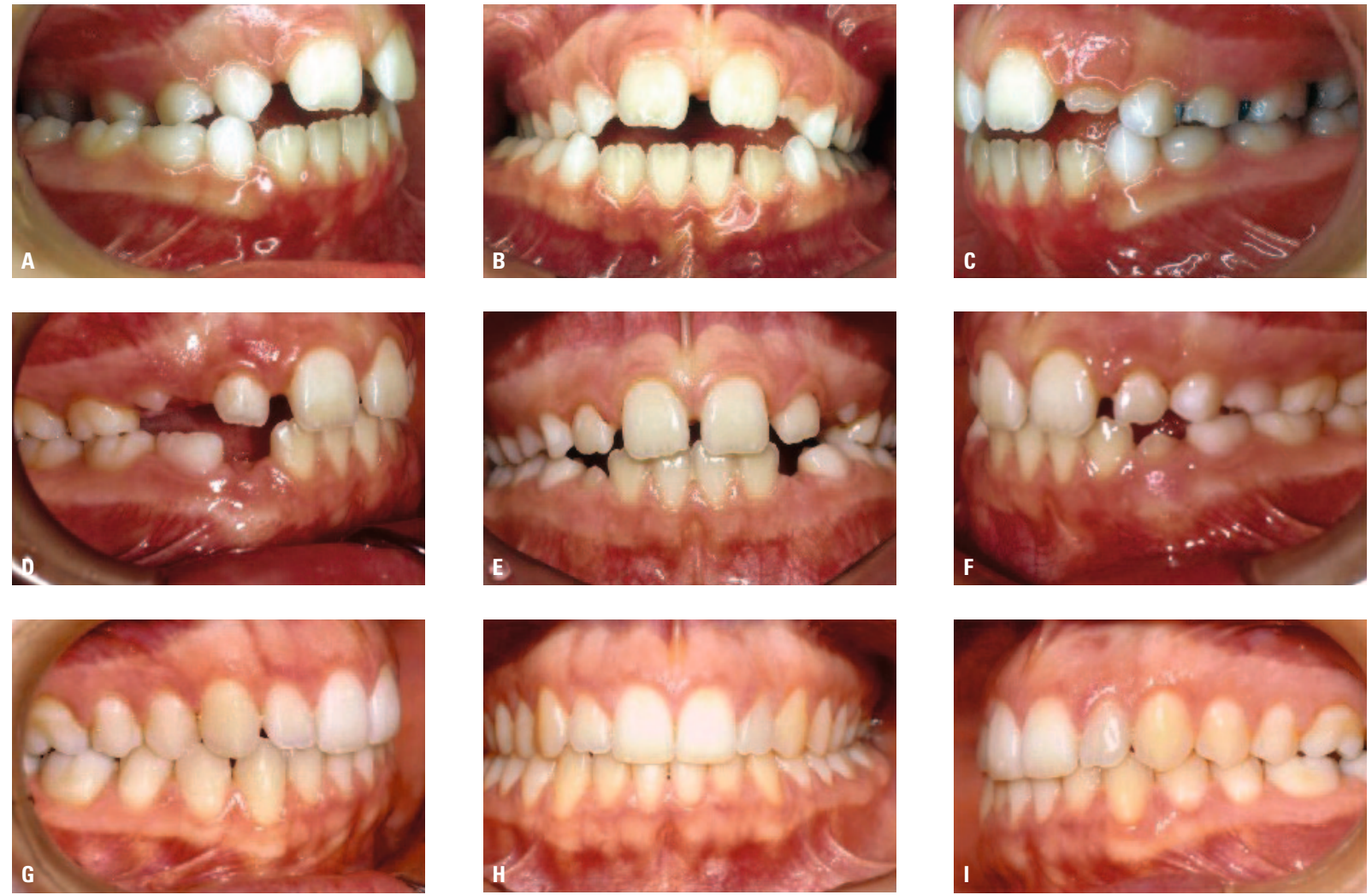

FIGURA 14 - Paciente portador de má oclusão de Classe II ilustrando a correção espontânea da discrepância anteroposterior após a ERM, nas seguintes fases: A, B, C) pré-tratamento, D, E, F) progresso e G, H, I) dentadura permanente.
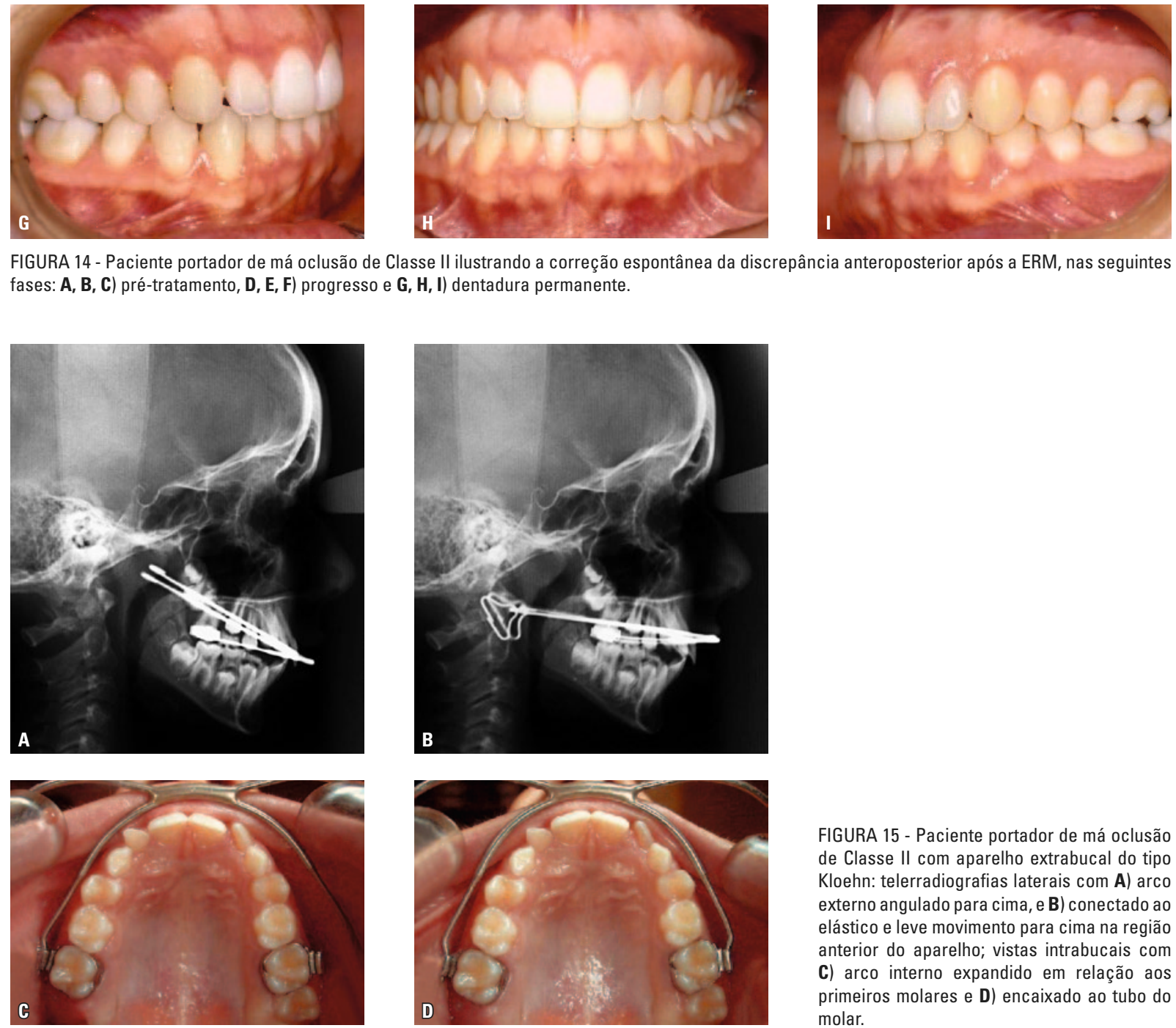
. 
devido ao estímulo do crescimento nesses locais e aos ajustes compensatórios em todas as outras suturas maxilocranianas.

As consequências dos benefícios diretos e indiretos do uso de aparelho extrabucal propiciam equilíbrio dos músculos mastigatórios e bucofaciais por meio do crescimento, conduzindo ao principal objetivo do tratamento ortodôntico, que é a estabilidade do resultado final.

No tratamento ortodôntico é fundamental que os elementos funcionais e estéticos das relações dentárias e esqueléticas que necessitam de correção sejam reconhecidos. O diagnóstico correto e individualizado para cada paciente possibilita a elaboração de um tratamento mais apropriado.

A expansão ortopédica da maxila vem atraindo, cada vez mais, a atenção da comunidade científica, devido principalmente à sua aplicação e capacidade de alterar o crescimento craniofacial em diversas situações clínicas. Esse tipo de intervenção ortopédica possui grande utilidade terapêutica, pois sua aplicação em diversas anormalidades apresenta maior versatilidade quando comparada aos aparelhos de modificação de crescimento disponíveis atualmente para o tratamento ortodôntico.

\title{
Transverse dimension alterations using rapid maxillary expansion
}

\begin{abstract}
Transverse deformities, typically manifested by unilateral or bilateral crossbite, are the skeletal problems that can cause more sequels in the craniofacial region. However, such deformities are the most adaptable to orthopedic changes. Rapid maxillary expansion has become a routine in the orthodontic practice. Even though initially such procedure has been used for correction of posterior crossbite, today it has been applied for indirect expansion of the lower arch, obtaining space for correction of dental crowding, correction of axial inclination of posterior teeth, improvement in the smile aesthetics characteristics and on treatment of Class II patients. Orthopedic maxillary expansion has gained increasing attention of the scientific community due to its application and ability to change the craniofacial growth in many clinical situations. This type of orthopedic intervention has a positive therapeutic result in several abnormalities and is more versatile when compared to the growth modification devices currently available for orthodontic treatment.
\end{abstract}

Keywords: Transverse dimension. Rapid maxillary expansion. Class II malocclusion.

\section{REFERÊNCIAS}

1. ADKINS, M. D.; NANDA, R. S.; CURRIER, G. F. Arch perimeter changes on rapid palatal expansion. Am. J. Orthod. Dentofacial Orthop., St. Louis, v. 97, no. 3, p. 194-199, 1990.

2. BACCETTI, T.; FRANCHI, L.; MCNAMARA, J.; TOLLARO, I. Early dentofacial features of Class II malocclusion: A longitudinal study from the deciduous through the mixed dentition. Am. J. Orthod. Dentofacial Orthop., St. Louis, v. 111, no. 5, p. 502-509, May 1997.

3. BRODIE, A. G. Anatomy and physiology of head and neck musculature. Am. J. Orthod., St. Louis, v. 36, no. 11, p. 831-844, 1950.
4. BRODIE, A. G. Consideration of musculature in diagnosis, treatment and retention. Am. J. Orthod., St. Louis, v. 38, no. 11, p. 823-835, Nov. 1952.

5. EVANS, C. A. Anteroposterior skeletal change: Growth modification. Semin. Orthod., Philadelphia, v. 6, no. 1, p. 21-32, Mar. 2000.

6. FRANCHI, L.; BACCETTI, T. Transverse maxillary deficiency in Class II and Class III malocclusions: A cephalometric and morphometric study on postero-anterior films. Orthod. Craniofac. Res., Oxford, v. 8, no. 1, p. 21-28, Feb. 2005. 
7. GIANELLY, A. A. Bidimensional technique: Theory and practice. New York: GAC International, 2000.

8. HAAS, A. J. Andrew J. Haas: entrevista. R. Dental Press Ortodon. Ortop. Facial, Maringá, v. 6, n. 1, p. 1-10, jan./fev. 2001.

9. HAAS, A. J. Headgear therapy: The most efficient way to distalize molars. Semin. Orthod., Philadelphia, v. 6, no. 2 p. 79-90, June 2000.

10. HAAS, A. J. Long-term posttreatment evaluation of rapid palatal expansion. Angle Orthod., Appleton, v. 50, no. 3, p. 189-217, 1980.

11. HAAS, A. J. Palatal expansion: Just the beginning of dentofacial orthopedics. Am. J. Orthod., St. Louis, v. 57, no. 3, p. 219-255, Mar. 1970.

12. HOWE, R. P.; McNAMARA, J. A.; O'CONNOR, K. A. An examination of dental crowding and its relationship to tooth size and arch dimension. Am. J. Orthod., St. Louis, v. 83, no. 5, p. 363-373, 1983.

13. KIRJAVAINEN, M.; KIRJAVAINEN, T. Maxillary expansion in Class II correction with orthopedic cervical headgear: A posteroanterior cephalometric study. Angle Orthod., Appleton v. 73, no. 3, p. 281-285, June 2003.

14. KIRJAVAINEN, M.; KIRJAVAINEN, T.; HAAVIKKO, K. Changes in dental arch dimensions by use of an orthopedic cervical headgear in Class II correction. Am. J. Orthod. Dentofacial Orthop., St. Louis, v. 111, no. 1, p. 59-66, Jan. 1997.

15. KIRJAVAINEN, M.; KIRJAVAINEN, T.; HURMERINTA, K.; HAAVIKKO, K. Orthopedic cervical headgear with an expanded inner bow in Class II correction. Angle Orthod., Appleton, v. 70, no. 4, p. 317-325, Aug. 2000.

16. LIMA FILHO, R. M. A.; LIMA, A. L. Long-term outcome of Class II division 1 malocclusion treated with rapid palatal expansion and cervical traction. Angle Orthod., Appleton, v. 70, no. 1, p. 89-94, Feb. 2000

17. LIMA FILHO, R. M. A.; LIMA, A. L.; RUELLAS, A. C. O. Longitudinal study of anteroposterior and vertical maxillary changes in skeletal Class II patients treated with Kloehn cervical headgear. Angle Orthod., Appleton, v. 73, no. 2, p. 187-193, Apr. 2003.

18. LIMA FILHO, R. M. A.; RUELLAS, A. C. O. Long-term anteroposterior and vertical maxillary changes in skeletal Class II patients treated with slow and rapid maxillary expansion. Angle Orthod., Appleton, v. 77, no. 5, p. 870-874, Sept. 2007.
19. LIMA FILHO, R. M. A. RUELLAS, A. C. O. Mandibular behavior with slow and rapid maxillary expansion in skeletal Class II patients. Angle Orthod., Appleton, v. 77, no. 4, p. 625-631, July 2007.

20. LIMA, A. C.; LIMA, A. L.; LIMA FILHO, R. M. A.; OYEN, O. J. Spontaneous mandibular arch response after rapid palatal expansion: A long-term study on Class I malocclusion. Am. J. Orthod. Dentofacial Orthop., St. Louis, v. 126, no. 5, p. 576-582, Nov. 2004

21. LIMA, A. L.; LIMA FILHO, R. M. A.; BOLOGNESE, A. M. Longterm clinical outcome of rapid maxillary expansion as the only treatment performed in Class I malocclusion. Angle Orthod., Appleton, v. 75, no. 3, p. 416-420, May 2005.

22. LIMA, R. M. A.; LIMA, A. L. Long-term outcome of Class II division 1 malocclusion treated with rapid palatal expansion and cervical traction. Angle Orthod., Appleton, v. 70, no. 1, p. 89-94, Feb. 2000.

23. McNAMARA, J. A.; BRUDON, W. L. Orthodontic and orthopedic treatment in the mixed dentition. Ann Arbor: Needham Press, 1993

24. SANDSTROM, R. A.; KLAPPER, L.; PAPACONSTANTINOU, S Expansion of the lower arch concurrent with rapid maxillary expansion. Am. J. Orthod. Dentofacial Orthop., St. Louis, v. 94, no. 4, p. 296-302, Oct. 1988.

25. SARVER, D. M. Esthetic Orthodontics and Orthognathic Surgery. St. Louis: Mosby Year Book, 1998.

26. STALEY, R.; STUNTZ, W. R.; PETERSON, L. C. A comparison of arch widths in adults with normal occlusion and adults with Class II, division 1 malocclusion. Am. J. Orthod., St. Louis, v. 88 , no. 2 , p. 163-169, 1985

27. TOLLARO, I.; BACCETTI, T.; FRANCHI, L.; TANASESCU, C. D. Role of posterior transverse interarch discrepancy in Class II division 1 malocclusion during the mixed dentition phase. Am. J. Orthod. Dentofacial Orthop., St. Louis, v. 110, no. 4, p. 417-422, Oct. 1996

28. WILL, L. A. Transverse maxillary deformities: Diagnosis and treatment. J. Oral Maxillofac. Surg., Philadelphia, v. 5, p. $1-28,1996$
Endereço para correspondência

Roberto Mario Amaral Lima Filho

Avenida Alberto Andaló 4025

CEP: 15.015-000 - São José do Rio Preto / SP

E-mail: robertomario@terra.com.br 